\title{
Images of the month: An incidental finding of spontaneous pneumomediastinum (Hamman's syndrome) secondary to diabetic ketoacidosis during the coronavirus pandemic
}

\author{
Authors: Keisha A Marchon, ${ }^{\mathrm{A}}$ Maya O Nunn ${ }^{\mathrm{B}}$ and Ali J Chakera ${ }^{\mathrm{C}}$
}

\begin{abstract}
Hamman's syndrome is a spontaneous pneumomediastinum and is described as a rare complication of diabetic ketoacidosis (DKA). It typically follows a self-limiting course after successful treatment of the underlying DKA. We describe a case of a 28-year-old woman with type 1 diabetes presenting with facial pain, vomiting and abdominal pain. She also complained of dyspnoea and chest pain. She was diagnosed and treated for DKA triggered by a dental abscess. Given the presentation during the coronavirus pandemic, a computed tomography pulmonary angiography was performed in line with the diagnostic pathway for COVID-19, which incidentally showed a significant pneumomediastinum and pneumopericardium. The patient was initially investigated for oesophageal rupture secondary to vomiting (Boerhaave's syndrome), however, remained clinically stable throughout. Follow-up computed tomography showed near-complete resolution of pneumomediastinum with no intervention other than treatment of DKA. This therefore confirmed Hamman's syndrome.

We propose that given the benign nature of the condition and the incidental finding in this report, as well as poor identification of mediastinal gas on chest X-ray, Hamman's syndrome is more common than reported.
\end{abstract}

KEYWORDS: Diabetic ketoacidosis, type 1 diabetes mellitus, pneumomediastinum, Hamman's syndrome

DOI: $10.7861 /$ clinmed.2020-0739

\section{Case presentation}

A 28-year-old woman with type 1 diabetes was admitted with vomiting, left-sided facial pain and intermittent abdominal pain. She described pleuritic chest pain and shortness of breath. She

Authors: ${ }^{{ }}$internal medicine training year-2 doctor, Royal Sussex County Hospital, Brighton, UK; ${ }^{B}$ acute care common stem year-1 doctor, Royal Sussex County Hospital, Brighton, UK; ' ${ }^{\text {C consultant }}$ in diabetes and endocrinology, Royal Sussex County Hospital, Brighton, UK and Brighton and Sussex Medical School, Brighton, UK was alert. There were crackles on auscultation in the right lower chest zone. There was a large firm swelling, consistent with a dental abscess on the left side of the face. Oxygen saturation was $100 \%$ on room air. All other bedside observations were normal. Venous blood gas showed $\mathrm{pH}$ 7.0; glucose at $22.3 \mathrm{mmol} / \mathrm{L}$; bicarbonate at $3.2 \mathrm{mmol} / \mathrm{L}$; and capillary blood ketones were $3.8 \mathrm{mmol} / \mathrm{L}$. Abnormal blood results included white cell count $\left(16.5 \times 10^{9} / \mathrm{L}\right.$; neutrophils $14.5 \times 10^{9} / \mathrm{L}$; and lymphocytes $\left.0.7 \times 10^{9} / \mathrm{L}\right)$, C-reactive protein (35 mg/L) and alkaline phosphatase (147 IU/L). Her glycated haemoglobin was $117 \mathrm{mmol} / \mathrm{mol}$ (12.9\%).

The working diagnosis was diabetic ketoacidosis (DKA) secondary to a dental abscess. She was commenced on antibiotics, fixed-rate insulin infusion and fluid resuscitation. Given the coronavirus pandemic and presentation with dyspnoea, COVID-19 was a differential.

The patient's chest X-ray was reported as normal (Fig 1a). The subsequent contrast-enhanced computed tomography (CT) showed an extensive pneumomediastinum and pneumopericardium of unclear cause (Figs $1 \mathrm{~b}$ and c). Her swab was negative for SARS-CoV-2.

The patient was managed as having oesophageal rupture secondary to vomiting (Boerhaave's syndrome), with total parenteral nutrition, on intensive care. DKA resolved within a few hours of admission, euglycaemia was maintained with variablerate intravenous insulin. Maxillofacial surgeons treated an acute apical left lower molar (LL6) abscess with antibiotics and planned elective extraction. By day 5 of admission, the patient's symptoms had resolved.

The patient remained clinically stable, out of keeping with typical Boerhaave's syndrome; a diagnosis of Hamman's syndrome was suggested. Follow-up CT (Fig 2) showed significantly improved appearances of the pneumomediastinum and no evidence of oesophageal rupture. These features were key in confirming the suspicion of Hamman's syndrome. The patient was discharged on her usual insulin regimen.

\section{Discussion}

This report presents a rare diagnosis, which was identified due to the diagnostic pathways used during the coronavirus pandemic. Physiologically, pressure in the mediastinum is lower than alveolar pressure. In DKA, alveolar rupture causes the movement 
Fig 1. Imaging on admission. a) Chest $X$-ray reported as normal, with subtle signs of pneumomediastinum (red arrow). b and c) Contrast enhanced computed tomography of the thorax showing extensive pneumomediastinum and pneumopericardium.
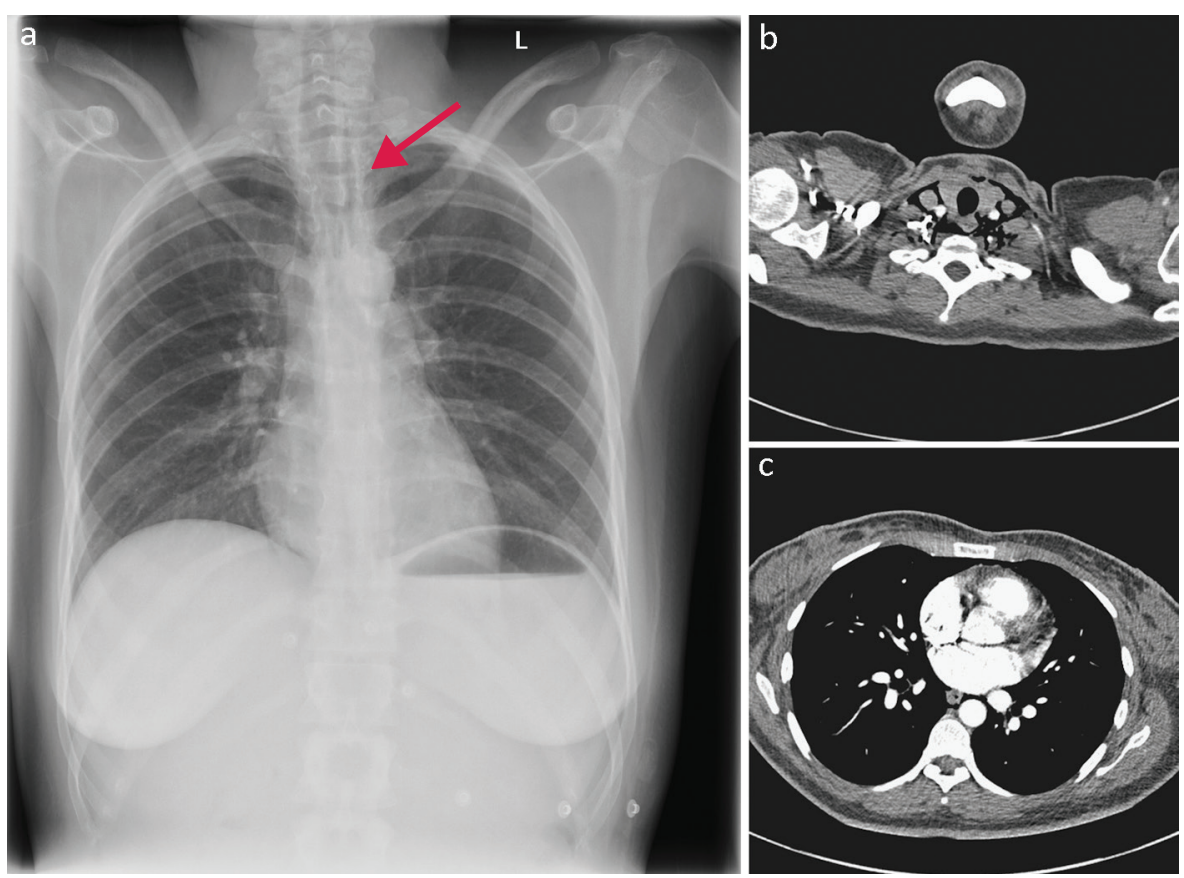

of air down the pressure gradient into the mediastinal space. ${ }^{1-4}$ Alveolar rupture in DKA is thought to be precipitated by Kussmaul breathing and severe vomiting. ${ }^{1}$ The most common symptoms are chest pain and dyspnoea.

In the majority of 79 reported cases, after correction of acidosis and vomiting, pathological collections of air were gradually reabsorbed. ${ }^{1}$ The benign, self-limiting course of Hamman's syndrome is a reason we infer that many cases remain undiagnosed, a theory proposed in other literature. ${ }^{3}$ Two fatal cases have been described due to worsening pneumomediastinum and another due to pulmonary infection. ${ }^{4,5}$

We hypothesise that outside of the coronavirus pandemic, this patient would have been successfully treated for DKA and the pneumomediastinum and pneumopericardium would have remained undiagnosed. As this diagnosis was identified on CT that formed part of the COVID-19 investigation pathway, this implies that Hamman's syndrome may be more common than presumed.
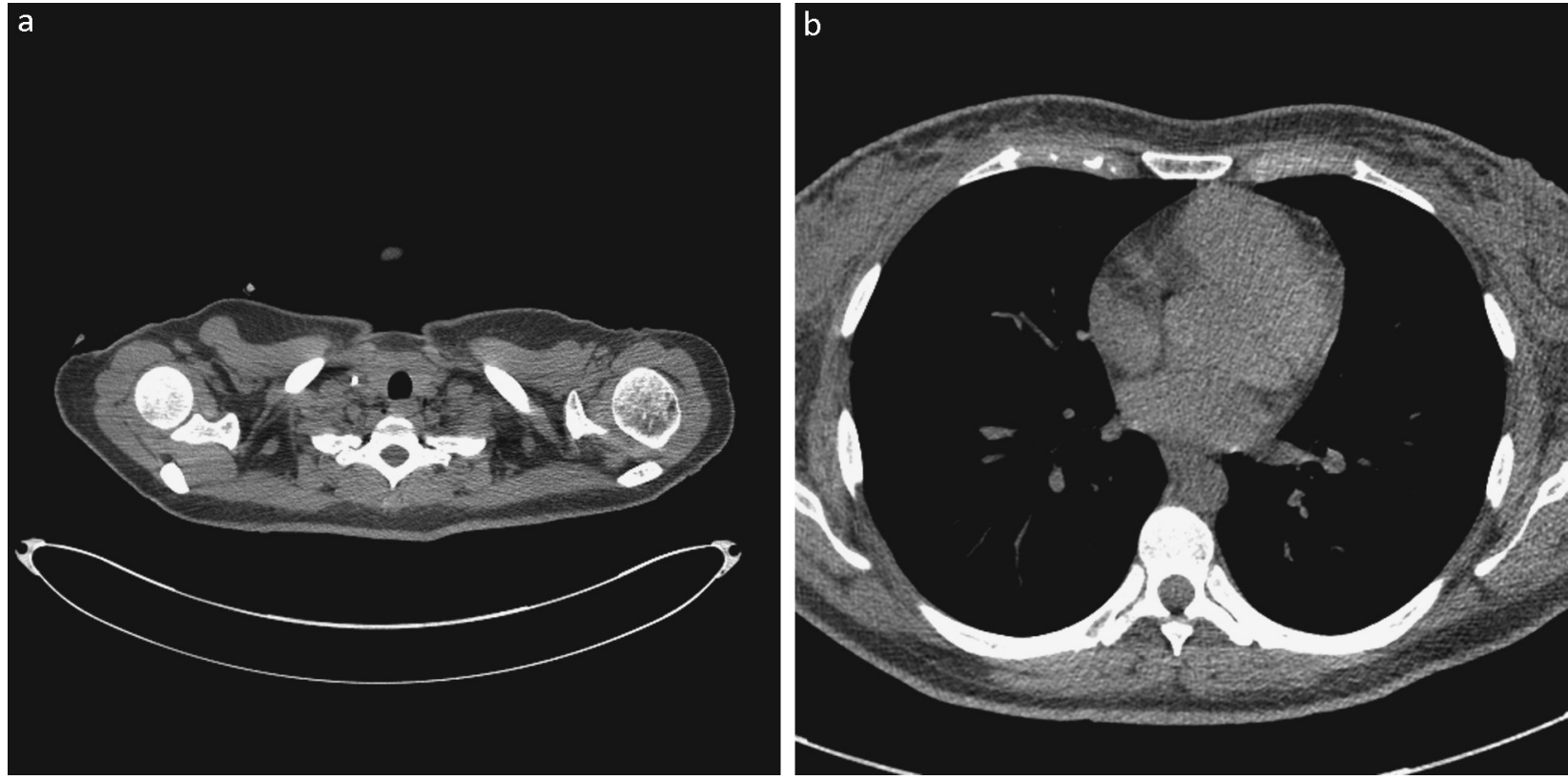

Fig 2. Computed tomography of the thorax on day 7 of admission showing resolved pneumomediastinum and pneumopericardium. 


\section{Acknowledgements}

We would like to thank the patient for consenting to allow us to write this case report and the multidisciplinary team involved in her care.

\section{References}

1 Zhang W, Chen J, Wu X et al. Analysing the clinical features of pneumomediastinum associated with diabetic ketoacidosis in 79 cases. Diabetes Metab Syndr Obes 2020;13:405.

2 Pain AR, Pomroy J, Benjamin A. Hamman's syndrome in diabetic ketoacidosis. Endocrinol Diabetes Metab Case Rep 2017;2017: 17-0135.
3 Girard DE, Carlson V, Natelson EA, Fred HL. Pneumomediastinum in diabetic ketoacidosis: comments on mechanism, incidence, and management. Chest 1971;60:455-9.

4 Ruttley M, Mills RA. Subcutaneous emphysema and pneumomediastinum in diabetic keto-acidosis. Br J Radiol 1971;44:672-4.

5 Shang J, Yao HB, Zhang YB. Pneumomediastinum in a patient with diabetic ketoacidosis: one case report. Tianjin Medical Journal 2011;8:691.

Address for correspondence: Dr Ali Chakera, Royal Sussex County Hospital, Brighton and Sussex University Hospitals NHS Trust, Eastern Road, Brighton BN2 5BE, UK.

Email: alichakera@nhs.net 